Research Paper

\title{
A combined marker based on plasma D-dimer and serum albumin levels in patients with nasopharyngeal carcinoma is associated with poor survival outcomes in a retrospective cohort study
}

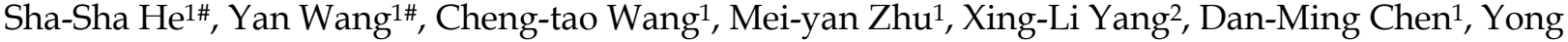 \\ Chen ${ }^{1 凶}$ \\ 1. Department of Radiation Oncology, The First Affiliated Hospital of Sun Yat-Sen University, Guangzhou, Guangdong 510080, PR China. \\ 2. Department of Radiation Oncology, Sun Yat-Sen University Cancer, Guangzhou, Guangdong 510060, PR China. \\ \#These authors contributed equally to this work. \\ $\triangle$ Corresponding author: Yong Chen, M.D. Department of Radiation Oncology, The First Affiliated Hospital of Sun Yat-Sen University, Guangzhou, \\ Guangdong 510080, PR China. Telephone: +0086-020-87755766, E-mail: chenyong@mail.sysu.edu.cn \\ (c) Ivyspring International Publisher. This is an open access article distributed under the terms of the Creative Commons Attribution (CC BY-NC) license \\ (https://creativecommons.org/licenses/by-nc/4.0/). See http://ivyspring.com/terms for full terms and conditions.
}

Received: 2018.12.18; Accepted: 2019.05.10; Published: 2019.06.09

\begin{abstract}
Background: Activation of the clotting-fibrinolytic system in cancer patients is common and results in an unfavorable clinical outcome. This study aimed to investigate the role of pretreatment plasma D-dimer levels and the combination of D-dimer and albumin (DA) on the prediction of survival prognosis in patients with nasopharyngeal carcinoma (NPC).

Methods: The study comprised 511 patients with NPC. Pretreatment plasma D-dimer and serum albumin levels were measured. DA was classified as a new biomarker where D-dimer and albumin levels were combined and was grouped by the cutoff value of both. The correlations of plasma D-dimer levels with clinicopathological features and survival outcome were calculated using the Chi-square test. Kaplan-Meier estimates were performed to analyze the survival functions and were compared using log-rank tests. Cox proportional hazard regression analysis was used to assess the effects of D-dimer and DA on distant overall survival (OS) and distant metastasis-free survival (DMFS).

Results: The median follow-up period was 45.2 months (range 2.1-79.8). Elevated plasma D-dimer levels were positively associated with age at diagnosis $(P=0.034)$, platelet levels $(P=0.043)$, and Epstein Barr Virus $(E B V)$ DNA copy number $(P=0.035)$. Additionally, multivariate analysis demonstrated that elevated plasma D-dimer levels were strongly associated with a poorer OS (HR 2.074, 95\% Cl 1.190-3.612, $P=$ 0.010), but not DMFS. After adjustment for other variables, DA stratification acted as an independent prognostic marker for OS $(P=0.038)$ and DMFS $(P=0.031)$ in patients with NPC, when combined with albumin levels.

Conclusions: Increased plasma D-dimer levels accurately predict poor OS and may be an effective independent prognostic factor in patients with NPC. Moreover, in conjunction with serum albumin, DA may serve as a factor in predicting OS and DMFS.
\end{abstract}

Key words: Nasopharyngeal carcinoma; D-dimer; albumin; prognosis

\section{Introduction}

Nasopharyngeal carcinoma (NPC) is a prevalent form of cancer in men aged between 20 and 44 years in parts of Southeast Asia (1). Furthermore, it is prevalent in southern China, especially Guangdong province (2), where it has even been termed "Canton tumor", and affects a significant proportion of the population in these geographically higher risk areas. The Canton-style diet is regarded as an important 
modifier of the NPC endemic and may partially account for the geographic distribution of NPC incidence (3). Patients with undifferentiated NPC, which accounts for $90 \%$ of cases in endemic regions, are generally sensitive to radiation and chemotherapy (4). Radiotherapy with or without chemotherapy is therefore the mainstream choice of treatment for primary NPC. Currently, modern anticancer strategies have provided opportunities to improve local control and survival in NPC (5). Precision radiotherapy techniques have emerged including three-dimensional conformal radiotherapy (3DCRT), intensity-modulated radiation therapy (IMRT), and stereotactic radiosurgery (SRS). The regimens of neoadjuvant chemotherapy based mainly on cisplatin, in combination with paclitaxel, docetaxel, 5-fluorouracil, and gemcitabine have shown encouraging results in NPC (6). Novel therapies targeting the epidermal growth factor receptor (EGFR) including nimotuzumab (7) and autologous cytotoxic T lymphocytes targeting EBV (8) have the potential to obtain optimal outcomes with minimal toxicity to the patient. Outcomes in patients with NPC have improved mainly because of these advances in comprehensive therapy strategies. However, although the currently established therapy for NPC has significantly improved, half of patients will experience local regional failure and distant metastasis during follow-up (9). It has demonstrated that recurrent head and neck tumors might be intrinsically more radioresistant than the primary tumors. Radiation can induce tissue fibrosis and microvasculature damage, while altering the tumor microenvironment by stimulating hypoxia status, resulting in significant difficulties for treatment (10). Treating recurrent NPC is always a huge challenging clinical problem, inducing limited local control. Further individualized management based on existing biomarkers should be analyzed more deeply.

In recent years, much attention has been given to the correlation between tumor development, progression and metastasis, and the coagulation and fibrinolysis system. Cancer-associated thrombosis significantly affects the lives of patients to their detriment. Indeed, research data suggests that targeting the coagulation system or fibrinolysis system might influence the course of malignant disease (11). The D-dimer is known as a degradation product of fibrin, which is one of the signals that activates the fibrinolytic system, and appears in the blood as a small peptide fragment (12). In most tumors, elevated D-dimer levels indicate a more advanced stage and increased risk for death (13). They have been evidenced in progression and metastasis (14) in malignancies such as lung cancer (15), gastric cancer (16), ovarian cancer (17), esophageal cancer (18), and NPC (19). Furthermore, it has been revealed that plasma D-dimer levels may be helpful in evaluating the response to chemotherapy in patients with metastatic colorectal cancer (20).

Therefore, in the present study, we aimed to explore the association of pretreatment plasma D-dimer levels with clinical and pathological factors as well as the treatment outcomes in patients with NPC. In addition, we attempted to combine the pretreatment albumin level to further assess the prognostic value of the both.

\section{Materials and Methods}

\section{Patients}

Demographic and clinical data from 511 patients who underwent radiotherapy between January 2010 and October 2013 for NPC were evaluated in this retrospective study. We routinely collected the laboratory testing data before treatment (plasma D-dimer, Epstein-Barr virus (EBV), serum albumin, and platelet levels). The staging affairs included a complete medical history, physical examination, direct re-optic nasopharyngoscopy, nasopharyngeal and neck magnetic resonance imaging (MRI), abdominal ultrasound, chest radiography and entire body bone scans, with or without positron emission tomography-computed tomography (PET-CT). The patients were restaged using the 8th edition of the International Union against Cancer/American Joint Committee on Cancer (UICC/AJCC) (21).

This study was approved by the Research Ethics Committee of Sun Yat-sen University Cancer Center, Guangzhou, China. The inclusion criteria for this study were as follows: (1) newly pathologically diagnosed primary NPC, (2) no history of previous anticancer therapy and first received radiotherapy with or without chemotherapy in our hospital, and (3) adequate clinical information in the medical record. Patients were excluded from the study for the following reasons: (1) venous or arterial thromboembolism within the preceding 3 months, (2) continuous anticoagulation with vitamin $\mathrm{K}$ antagonists (VKAs) or low-molecular-weight heparins, (3) known congenital thrombophilia, ongoing anticoagulant treatment, pregnancy, stroke or neurosurgery within 6 months, and (4) had developed metastasis at diagnosis.

\section{Treatment}

In our study, a standard multi-therapeutic protocol was used to treat the patients with NPC. All patients received intensity-modulated radiation therapy (IMRT), while radiation therapy alone was used for stage I patients, and radiation with 
concurrent platinum-based chemotherapy was used to treat stage II patients. Concurrent chemoradiotherapy, with or without neoadjuvant or adjuvant chemotherapy, was used for advanced stage NPC (stages III and IVA-B). Neoadjuvant or adjuvant chemotherapy consisted of cisplatin with 5-fluorouracil or cisplatin with taxane, administered every 3 weeks for 2 or 3 cycles. The concurrent chemotherapy regimen consisted of cisplatin administered on weeks 1,4 , and 7 of radiotherapy, or weekly. All patients at the study institution were treated according to the principle of treatment for NPC patients at Sun Yat-sen University Cancer Center, Guangzhou, China. It had been clarified in our previously published paper (22).

\section{Measurement of D dimer levels}

In all patients, peripheral blood samples were collected within 1 week before treatment. The levels of D-dimer were measured using a nephelometry immunoassay (Axis-Shield PoC AS, Norway) at our hospital. We used a cutoff level of $0.675 \mu \mathrm{g} / \mathrm{ml}$ to distinguish between a high and low group according to the ROC curve analysis. Furthermore, other pretreatment laboratory data including EBV DNA and platelet levels were also collected from medical records in our study. The median albumin value was $45 \mathrm{~g} / \mathrm{L}$. Accordingly, the cutoff values of $\mathrm{D}$ dimer and albumin were defined as $0.675 \mu \mathrm{g} / \mathrm{ml}$ and $45 \mathrm{~g} / \mathrm{L}$, respectively. Patients were distributed into three groups: DA group 1 represented low $\mathrm{D}$ dimer levels $(<0.675 \mu \mathrm{g} / \mathrm{ml})$ and high albumin levels $(\geq 45 \mathrm{~g} / \mathrm{L})$; high $\mathrm{D}$ dimer levels and high albumin levels, or low $\mathrm{D}$ dimer levels and low albumin levels were assigned DA group 2; DA group 3 was defined as that with high $\mathrm{D}$ dimer levels and low albumin levels.

\section{Follow-up}

Follow-up evaluations were performed every 3 months within the first 3 years, 6 months in the following 3-5 years, and annually thereafter until death. The surveillance strategy following treatment included physical examination, nasopharyngoscopy, nasopharyngeal and neck MRI, chest CT, abdominal ultrasonography, bone marrow scan (if any symptoms of bone metastasis), EBV DNA copy load, and monitoring of other haematological measurements.

Follow-up was measured from date of first diagnosis to either the date of death or loss to follow-up. Our primary endpoint was overall survival (OS). Distant metastasis-free survival (DMFS) was included as a secondary endpoint in this study. OS was calculated from the date of the first NPC diagnosis to the date of death from any cause or patient censoring at the date of the last follow-up. DMFS was determined from the date of the first NPC diagnosis to the date of distant relapse or patient censoring at the date of the last follow-up.

\section{Statistical analysis}

Receiver operating curve (ROC) analyses were used to determine the optimal cutoff values for the continuous plasma D-dimer, EBV DNA, and serum albumin levels. The Chi-square test was used to analyze the associations between pretreatment plasma D-dimer and clinicopathological features. The survival outcomes were analyzed using the Kaplan-Meier method, and comparisons were made using the log-rank test. Variables that reached a $p$-value of $\leq 0.05$ in the univariate analysis were entered into multivariate analyses to examine their independent prognostic value on survival outcomes. The hazard ratios (HRs) with 95\% confidence intervals (CI) were reported as relative risks for each independent factor. Statistical analysis was performed using the IBM SPSS statistical program package, version 22.0, IBM Corp. All statistical tests were two-sided; $P<0.05$ was defined as being statistically significant.

\section{Results}

\section{Correlations between Plasma Levels of D-Dimer and Other Clinical Characteristics}

A total of 511 patients with NPC who met our determined criteria were included in this study. Clinicopathologic characteristics and plasma D-dimer levels of the patients are summarized in Table 1 . In total, 338/511 (66.1\%) patients had a pretreatment plasma D-dimer level below the cutoff value $(0.675$ $\mathrm{ug} / \mathrm{ml})$ and $173 / 511(33.9 \%)$ were above the cutoff value. The median age of the 511 patients was 45 years. According to the analysis, the elevated D-dimer levels were significantly correlated with older age $(P=$ $0.034)$, platelet levels $(P=0.043)$, and EBV DNA copy number $(P=0.035)$.

\section{High pretreatment plasma D-dimer is an independent prognostic factor for poor OS}

The median follow-up duration period was 45.2 months (range, 2.1-79.8). For the entire cohort of 511 patients, the 3-year overall survival rate was $92.1 \%$. In survival analysis, a high pretreatment plasma D-dimer level was associated with a poorer 3-year OS compared with a low pretreatment plasma D-dimer level (88.3\% vs. $93.6 \%$; Fig. $1 \mathrm{~A}$; log-rank test, $P=0.001)$ but DMFS was not significantly different (Fig.1B; $P=$ $0.381)$.

To determine the independent prognostic value of the plasma D-dimer level for OS and DMFS, 
multivariate analyses using a Cox proportional hazard model were performed to adjust for known prognostic parameters including smoking status, therapy regimen, overall stage, $\mathrm{T}$ category, $\mathrm{N}$ category, C-reactive protein, platelet levels, EBV DNA load, and D-dimer levels. The multivariate analyses demonstrated that a high pretreatment plasma D-dimer level was an independent prognostic factor for poor OS (HR 2.074, 95\% CI 1.190-3.612, $P=0.010$; Table 2) after adjustment for other factors.

Table 1: Association between D-dimer levels and clinicopathological features in patients with nasopharyngeal carcinoma $(n=511)$

\begin{tabular}{|c|c|c|c|c|}
\hline \multirow[t]{2}{*}{ Characteristic } & \multirow{2}{*}{$\begin{array}{l}\text { Total } \\
(\text { No, } \%)\end{array}$} & \multicolumn{2}{|c|}{ D-dimer $(\mu \mathrm{g} / \mathrm{ml})$} & \multirow[t]{2}{*}{$P$-value } \\
\hline & & $<0.675$ & $\geq 0.675$ & \\
\hline & $511(100)$ & $338(66.1)$ & $173(33.9)$ & \\
\hline Age (years) & & & & 0.034 \\
\hline$<45$ & $256(50.1)$ & $180(53.3)$ & $76(43.9)$ & \\
\hline$\geq 45$ & 255 (49.9) & $158(46.7)$ & $97(56.1)$ & \\
\hline Smoking & & & & 0.818 \\
\hline Yes & $151(29.5)$ & $101(29.9)$ & $50(28.9)$ & \\
\hline No & $360(70.5)$ & $237(70.1)$ & $123(71.1)$ & \\
\hline Overall stage ( $8^{\text {th }}$ edition) & & & & 0.663 \\
\hline $\mathrm{I}+\mathrm{II}$ & $154(30.1)$ & $104(30.8)$ & $50(28.9)$ & \\
\hline III+IV & 357 (69.9) & $234(69.2)$ & $123(71.1)$ & \\
\hline $\mathrm{T}$ category & & & & 0.403 \\
\hline $\mathrm{T} 1-2$ & 193(37.8) & $132(39.1)$ & $61(35.3)$ & \\
\hline T3-4 & $318(62.2)$ & $206(60.9)$ & $112(64.7)$ & \\
\hline $\mathrm{N}$ category & & & & 0.666 \\
\hline N0-1 & $381(74.6)$ & $250(74.0)$ & $131(75.7)$ & \\
\hline N2-3 & $130(25.4)$ & $88(26.0)$ & $42(24.3)$ & \\
\hline Treatment & & & & 0.373 \\
\hline IMRT & $109(21.3)$ & $76(22.5)$ & $33(19.1)$ & \\
\hline $\mathrm{CT}+\mathrm{IMRT}$ & $402(78.7)$ & $262(77.5)$ & $140(80.9)$ & \\
\hline Platelet $\left(\wedge 10^{9} / \mathrm{L}\right)$ & & & & 0.043 \\
\hline$<249$ & $329(64.4)$ & $228(67.5)$ & $101(58.4)$ & \\
\hline$\geq 249$ & $182(35.6)$ & $110(32.5)$ & $72(41.6)$ & \\
\hline C-reactive protein $(\mathrm{mg} / \mathrm{L})$ & & & & 0.148 \\
\hline$<3.86$ & $352(68.9)$ & $240(71.0)$ & $112(64.7)$ & \\
\hline$\geq 3.86$ & $159(31.1)$ & $98(29.0)$ & $61(35.63)$ & \\
\hline Albumin (g/L) & & & & 0.932 \\
\hline$<45$ & $297(58.1)$ & $196(58.0)$ & $101(58.4)$ & \\
\hline$\geq 45$ & $214(41.9)$ & $142(42.0)$ & 72 (41.6) & \\
\hline EBV DNA (copies/ml) & & & & 0.035 \\
\hline$<3775$ & $246(48.1)$ & $174(51.5)$ & 72 (41.6) & \\
\hline$\geq 3775$ & $265(51.9)$ & $164(48.5)$ & $101(58.4)$ & \\
\hline Outcomes & & & & \\
\hline
\end{tabular}

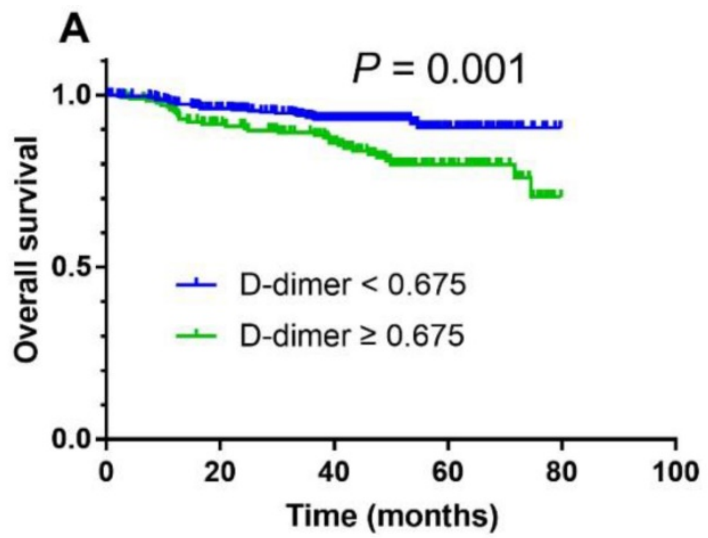

\begin{tabular}{llll}
\hline Characteristic & Total & D-dimer $(\boldsymbol{\mu g} / \mathrm{ml})$ & \multirow{2}{*}{ P-value $^{*}$} \\
\cline { 2 - 4 } & $\mathbf{( N o}, \mathbf{0})$ & $<\mathbf{0 . 6 7 5}$ & 31 \\
\hline Death & 55 & 24 & 23 \\
Distant metastasis & 67 & 44 & 3 \\
Localregional relapse & 17 & 14 & \\
\hline
\end{tabular}

Abbreviations: $\mathrm{IMRT}=$ intensity-modulated radiation therapy; $\mathrm{CT}=$ chemotherapy.

${ }^{*}$ Chi-square test

Table 2. Cox's proportional hazards regression model of overall survival for the 511 patients with nasopharyngeal carcinoma

\begin{tabular}{|c|c|c|c|c|}
\hline \multirow[t]{2}{*}{ Variable } & \multicolumn{2}{|l|}{ Univariate analysis } & \multicolumn{2}{|c|}{ Multivariate analysis } \\
\hline & HR $(95 \% \mathrm{CI})$ & $P$ & HR $(95 \%$ CI) & $P$ \\
\hline \multicolumn{5}{|l|}{ Smoking } \\
\hline Yes vs. no & $1.928(1.132-3.286)$ & 0.016 & $\begin{array}{l}2.191 \\
(1.264-3.796)\end{array}$ & 0.005 \\
\hline \multicolumn{5}{|l|}{ Therapy } \\
\hline IMRT+CT vs. IMRT & $2.299(1.326-3.985)$ & 0.003 & $\begin{array}{l}3.047 \\
(1.710-5.429)\end{array}$ & $<0.001$ \\
\hline \multicolumn{5}{|c|}{ Overall stage ( $8^{\text {th }}$ edition) } \\
\hline III+IV vs. I+II & $\begin{array}{l}9.117 \\
(2.844-29.227)\end{array}$ & $<0.001$ & & \\
\hline \multicolumn{5}{|l|}{$\mathrm{T}$ category } \\
\hline T3-4 vs. T1-2 & $\begin{array}{l}4.737 \\
(2.140-10.487)\end{array}$ & $<0.001$ & & \\
\hline \multicolumn{5}{|l|}{$\mathrm{N}$ category } \\
\hline N2-3 vs. N0-1 & $3.318(1.940-5.677)$ & $<0.001$ & $\begin{array}{l}2.838 \\
(1.556-5.177)\end{array}$ & 0.001 \\
\hline \multicolumn{5}{|l|}{$\begin{array}{l}\text { C-reactive protein } \\
(\mathrm{mg} / \mathrm{L})\end{array}$} \\
\hline$\geq 3.86$ vs. $<3.86$ & $2.261(1.331-3.841)$ & 0.003 & & \\
\hline \multicolumn{5}{|l|}{ Platelet $\left(\wedge 10^{9} / \mathrm{L}\right)$} \\
\hline$\geq 249$ vs. $<249$ & $1.992(1.173-3.383)$ & 0.011 & & \\
\hline $\begin{array}{l}\text { EBV DNA (copies/m } \\
\geq 3775 \text { vs. }<3775\end{array}$ & 3.177 (1.809-5.581) & $<0.001$ & $\begin{array}{l}3.621 \\
(2.010-6.523)\end{array}$ & $<0.001$ \\
\hline $\begin{array}{l}\text { D-dimer }(\mathrm{ug} / \mathrm{ml}) \\
\geq 0.675 \text { vs. }<0.675\end{array}$ & $2.423(1.417-4.142)$ & 0.001 & $\begin{array}{l}2.074 \\
(1.190-3.612)\end{array}$ & 0.010 \\
\hline $\mathrm{Abb}$ & ratio; $\mathrm{CI}=$ confid & nce inter & & \\
\hline
\end{tabular}

In combination with albumin, the DA group also had prognostic value in NPC. In univariate analysis, the 3-year OS rate in DA group 1 vs. group 2 vs. group 3 was $93.9 \%$ vs. $92.0 \%$ vs. $85.9 \%$ (Fig. 2 A; $P=0.002$ ), respectively. After adjusting for smoking status,

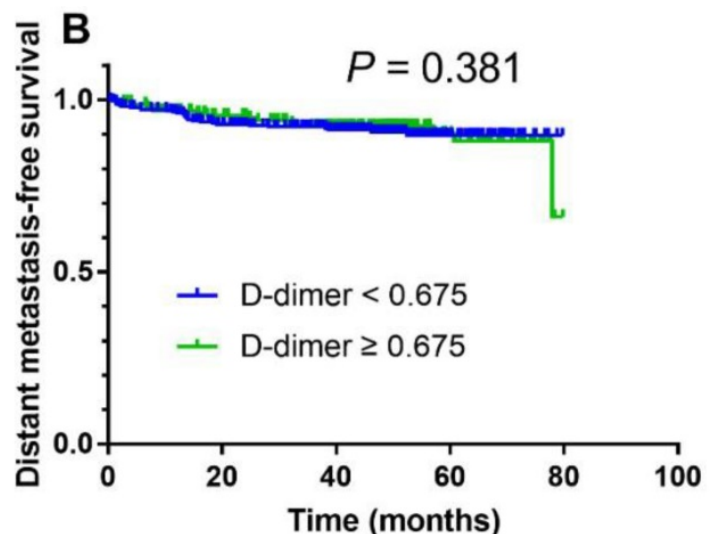

Figure 1. Kaplan-Meier overall survival (A) and distant metastasis-free survival (B) curves for all 511 patients with NPC stratified by pretreatment plasma D-dimer levels (< $0.675 \mu \mathrm{g} / \mathrm{ml}$ vs. $\geq 0.675 \mu \mathrm{g} / \mathrm{ml}$ ). 

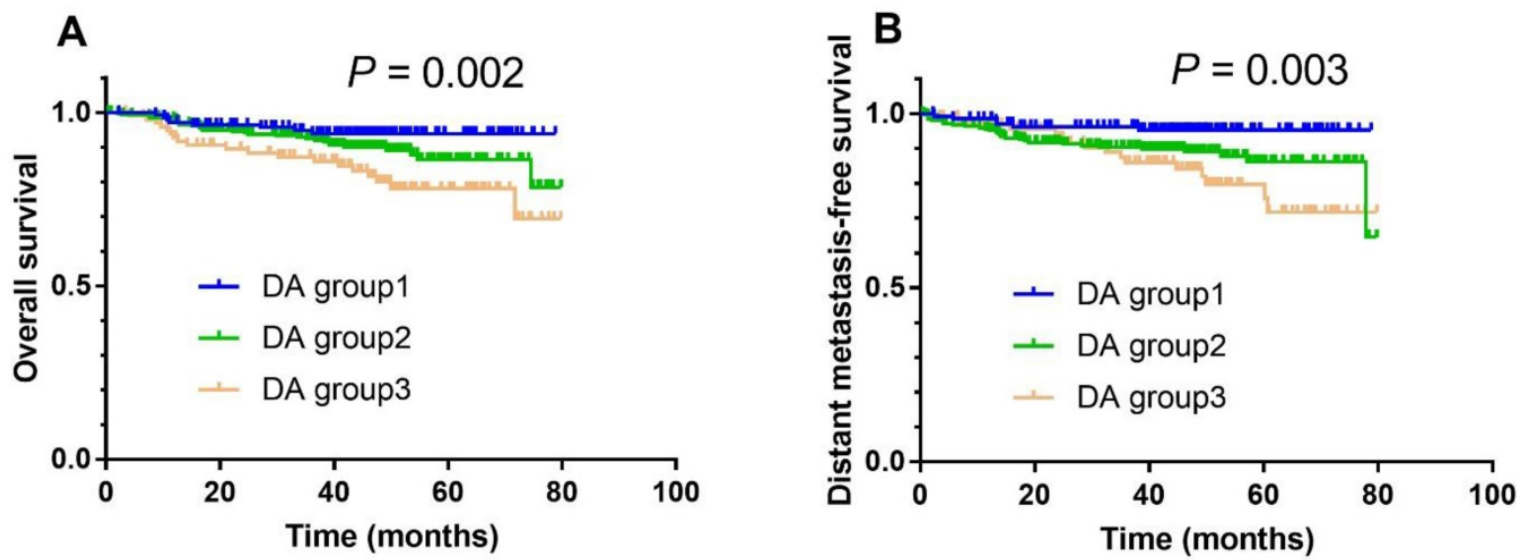

Figure 2. Kaplan-Meier overall survival (A) and distant metastasis-free survival (B) curves for all 511 patients with NPC stratified by DA stratification (DA group 1: D dimer levels $<0.675 \mu \mathrm{g} / \mathrm{ml}$ and albumin levels $\geq 45 \mathrm{~g} / \mathrm{L}$; DA group 2: D dimer levels $\geq 0.675 \mu \mathrm{g} / \mathrm{ml}$ and albumin levels $\geq 45 \mathrm{~g} / \mathrm{L}$, or D dimer levels $<0.675 \mu \mathrm{g} / \mathrm{ml}$ and albumin levels $<45$ $\mathrm{g} / \mathrm{L}$; DA group 3: D dimer levels $\geq 0.675 \mu \mathrm{g} / \mathrm{ml}$ and albumin levels $<45 \mathrm{~g} / \mathrm{L}$ ).

therapy regimen, overall stage, $\mathrm{N}$ category, C-reactive protein, and EBV DNA load, DA was shown to be an independent prognostic factor for OS (DA group $2 \mathrm{vs.}$ group 1; HR 1.560, 95\% CI 1.108-2.392, $P=0.041$; DA group 3 vs. group 1; HR 1.930, 95\% CI 1.145-3.251, $P=$ 0.014; Table 3). Meanwhile, the 3-year DMFS rate in DA group 1 vs. group 2 vs. group 3 was $94.2 \%$ vs. $91.2 \%$ vs. $86.6 \%$ (Fig. 2B; $P=0.003$ ), respectively. After adjusting for overall stage, $\mathrm{N}$ category, C-reactive protein, and EBV DNA load, DA was found to be an independent prognostic factor for DMFS (DA group 2 vs. group 1; HR 2.194, 95\% CI 1.127-4.272, $P=0.021$; DA group 3 vs. group 1; HR 2.141, 95\% CI 1.003-4.573, $P=0.049$; Table 3 ).

\section{Discussion}

The presented data demonstrate that the pretreatment D-dimer levels were significantly associated with OS. However, they had no independently prognostic value for DMFS. When combined with albumin levels, the DA group could act as an independent prognostic marker for OS and DMFS in patients with NPC. We believe this is a promising new biomarker.

Currently, much research has focused on the relationship between the activation of the hemostatic system and tumors, which includes the platelet/coagulation/fibrinolysis systems (11, 23, 24). Key steps in tumor progression, including cellular transformation, proliferation, tumor cell survival, and blood vessel formation (angiogenesis) can be mediated by components of these systems (25). These mechanisms have also been attributed to the stimulation of tumor adhesion (26), growth (27), and metastasis (28). The tumor microenvironment, which is influenced by hemostatic elements such as platelets, coagulation factors, fibrinogen and D-dimers are known as key factors in the multiple stages of cancer progression. Indeed, plenty of studies have been conducted to confirm the mechanism and associations with cancer.

Table 3. Multivariate analysis of overall survival and distant metastasis-free survival for the 511 patients with nasopharyngeal carcinoma

\begin{tabular}{|c|c|c|c|c|}
\hline \multirow[t]{2}{*}{ Variable } & \multicolumn{2}{|l|}{ OS } & \multicolumn{2}{|l|}{ DMFS } \\
\hline & $\mathrm{HR}(95 \% \mathrm{CI})$ & $P$ & $\mathrm{HR}(95 \% \mathrm{CI})$ & $P$ \\
\hline \multicolumn{5}{|l|}{ Smoking } \\
\hline Yes vs. no & $2.205(1.276-3.810)$ & 0.005 & & \\
\hline \multicolumn{5}{|l|}{ Therapy } \\
\hline IMRT+CT vs. IMRT & $2.924(1.644-5.199)$ & $<0.001$ & & \\
\hline \multicolumn{5}{|l|}{ Overall stage (8 $8^{\text {th }}$ edition) } \\
\hline III+IV vs. I+II & & & $3.145(1.102-8.973)$ & 0.032 \\
\hline \multicolumn{5}{|l|}{ N category } \\
\hline N2-3 vs. N0-1 & $2.690(1.474-4.909)$ & 0.001 & 1.989 (1.178-3.358) & 0.010 \\
\hline \multicolumn{5}{|l|}{ C-reactive protein $(\mathrm{mg} / \mathrm{L})$} \\
\hline$\geq 3.86$ vs. $<3.86$ & & & 1.987 (1.239-3.187) & 0.004 \\
\hline \multicolumn{5}{|l|}{ EBV DNA (copies/ml) } \\
\hline$\geq 3775$ vs. $<3775$ & $3.699(2.056-6.658)$ & $<0.001$ & $1.660(1.054-2.616)$ & 0.029 \\
\hline \multicolumn{5}{|l|}{ DA } \\
\hline DA group1 & 1 & 0.038 & 1 & 0.031 \\
\hline DA group 2 vs. DA group 1 & $1.560(1.108-2.392)$ & 0.041 & $2.194(1.127-4.272)$ & 0.021 \\
\hline DA group 3 vs. DA group 1 & $1.930(1.145-3.251)$ & 0.014 & $2.141(1.003-4.573)$ & 0.049 \\
\hline
\end{tabular}

Epithelial-mesenchymal transition (EMT), an important process during the onset of cancer cell migration, invasion, and metastatic dissemination, is also a core event in both tumorigenesis and developmental processes (29). Protease activated receptor-1 (PAR1), known as the coagulation factor II (thrombin) receptor (30), is activated by thrombin and then consequently affects epithelial to myofibroblast transition. A study (31) revealed that these effects were clearly prevented by a specific PAR-1 antagonist, indicating the interaction with thrombin and EMT. As such, PAR-1 targeting therapy may interfere with EMT. Other studies reported that thrombin increases invasive activity through the thrombin receptor and that inhibition of PAR-1 expression reduces the invasive potential of tumors (32). Additionally, vascular endothelial growth factor 
(VEGF), one of the critical factors in angiogenesis that contributes to metastasis, is upregulated by thrombin via PAR-1 in breast cancer cells (33). Thrombin may be involved in bone metastasis mediated by VEGF secretion and studies have demonstrated that inhibition of thrombin is of great value in suppression of tumor metastasis (34).

The fibrinolytic system has attracted attention as a possible mediator of cancer progression in recent years. It is generally accepted that plasma D-dimer is a stable fibrin degradation product. Plasma D-dimer has been studied for the evaluation of its prognostic value in various cancers, including NPC, and the findings were similar to our analysis, while our study had focused on the combination of both D-dimer and albumin. Also, all the NPC patients we recruited received intensity-modulated radiation therapy (IMRT), and there have no studied about this. Thus, this may be of some aspects of novelty. We found that D-dimer levels were significantly associated with OS but not DMFS. Innovatively, in our study, a new marker called DA, which combined D-dimer with albumin, was identified as an independent factor of OS and DMFS in patients with NPC. DA was also strongly associated with postoperative survival in esophageal squamous cell carcinoma patients (35). Therefore, DA may be a better and more precise indicator for the prognosis of patients with other cancers.

Though the present article provides a comprehensive overview of the mechanism of thrombin in cancer progression, and highlights the survival benefits that are indicated according to the stratification of hemostatic factors, other therapeutic approaches to cancer therapy have been developed by targeting platelets, coagulation, and fibrinolysis. In terms of hemostatic elements, this form of therapy is rarely in use. Although the currently established therapy for NPC includes effective IMRT and chemotherapy, given the high metastasis and recurrence rate, it is very important to identify prognostic factors for NPC that can help clinical decision-making. According to the results of our study, there is a hypothesis that reducing the D-dimer levels and improving the nutritious conditions may contribute to further survival benefit in NPC patients.

\section{Study limitations}

There were some limitations to our study. Firstly, our study was a retrospective study with asmall sample size. Secondly, the value of plasma D-dimer was measured only once before treatment. Therefore, the surveillance of dynamic changes associated with D-dimer is needed to demonstrate and confirm the aforementioned relationships.
Additionally, a ROC curve was commonly plotted to determine the cutoff value of plasma D-dimer for prognosis prediction. However, some studies selected the cutoff value according to the marginal value of the D-dimer. Thus, further sensitivity analysis is necessary to consider the various cutoff values. The third limitation was the short median follow-up time, and additional patient data will be reported after a longer follow-up duration. Furthermore, we cannot exclude that the patients with asymptomatic hematologic events could have influenced the accuracy of the D-dimer or impaired the disease course and patient survival.

\section{Conclusions}

In summary, based on the results of the current study, we conclude that plasma D-dimer and the combination of D-dimer and albumin (DA) were independent prognostic biomarkers in patients with NPC. Although we were not able to precisely determine the definite mechanism underlying the prognostic value of $\mathrm{D}$-dimer in this study, it is rational to suggest that patients with NPC with pretreatment increased D-dimer levels may need closer surveillance, and may even require adjuvant anti-fibrinolysis therapy.

\section{Acknowledgments}

This work was supported by the Natural Science Foundation of Guangdong Province, China [grant number 2016A020215083]. The funding agency had no role in the study design, data collection and analysis, decision to publish, or preparation of the manuscript.

\section{Competing Interests}

The authors have declared that no competing interest exists.

\section{References}

1. Parkin DM, Muir CS. Cancer Incidence in Five Continents. Comparability and quality of data. IARC scientific publications. 1992(120):45-173.

2. Wei WI, Sham JS. Nasopharyngeal carcinoma. Lancet (London, England). 2005;365(9476):2041-54

3. Jia WH, Luo XY, Feng BJ, Ruan HL, Bei JX, Liu WS, et al. Traditional Cantonese diet and nasopharyngeal carcinoma risk: a large-scale case-control study in Guangdong, China. BMC cancer. 2010;10:446.

4. Wee J. Nasopharyngeal cancer: a promising future. The Lancet Oncology. 2012;13(2):116-8.

5. Chan AT. Current treatment of nasopharyngeal carcinoma. European journal of cancer (Oxford, England : 1990). 2011;47 Suppl 3:S302-3.

6. Komatsu M, Arai Y, Yabuki K, Sano D, Shiono O, Sakuma Y, et al. Concurrent Chemoradiotherapy with Docetaxel, Cisplatin and 5-Fluorouracil (TPF) in Patients with Nasopharyngeal Carcinoma. Anticancer research. 2015;35(12):6861-7.

7. Wang F, Jiang C, Ye Z, Sun O, Liu T, Xu M, et al. Efficacy and safety of nimotuzumab with neoadjuvant chemotherapy followed by concurrent chemoradiotherapy for locoregionally advanced nasopharyngeal carcinoma. Oncotarget. 2017;8(43):75544-75556.

8. Khanna R, Busson P, Burrows SR, Raffoux C, Moss DJ, Nicholls JM, et al. Molecular characterization of antigen-processing function in nasopharyngeal carcinoma (NPC): evidence for efficient presentation of Epstein-Barr virus cytotoxic T-cell epitopes by NPC cells. Cancer research. 1998;58(2):310-4. 
9. Lee AW, Lin JC, Ng WT. Current management of nasopharyngeal cancer. Seminars in radiation oncology. 2012;22(3):233-44.

10. Langendijk JA, Bourhis J. Reirradiation in squamous cell head and neck cancer: recent developments and future directions. Current opinion in oncology. 2007;19(3):202-9.

11. Korte W. Changes of the coagulation and fibrinolysis system in malignancy: their possible impact on future diagnostic and therapeutic procedures. Clinical chemistry and laboratory medicine. 2000;38(8):679-92.

12. De Buyzere M, Philippe J, Duprez D, Baele G, Clement DL. Coagulation system activation and increase of D-dimer levels in peripheral arterial occlusive disease. American journal of hematology. 1993;43(2):91-4.

13. Ay C, Dunkler D, Pirker R, Thaler J, Quehenberger P, Wagner O, et al. High D-dimer levels are associated with poor prognosis in cancer patients. Haematologica. 2012;97(8):1158-64.

14. Seitz R, Rappe N, Kraus M, Immel A, Wolf M, Maasberg M, et al. Activation of coagulation and fibrinolysis in patients with lung cancer: relation to tumour stage and prognosis. Blood coagulation \& fibrinolysis : an international journal in haemostasis and thrombosis. 1993;4(2):249-54.

15. Ma X, Li Y, Zhang J, Huang J, Liu L. Prognostic role of D-dimer in patients with lung cancer: a meta-analysis. Tumour biology : the journal of the International Society for Oncodevelopmental Biology and Medicine. 2014;35(3):2103-9.

16. Liu L, Zhang X, Yan B, Gu Q, Zhang X, Jiao J, et al. Elevated plasma D-dimer levels correlate with long term survival of gastric cancer patients. PloS one. 2014;9(3):e90547.

17. Liu $\mathrm{P}$, Wang $\mathrm{Y}$, Tong L, Xu Y, Zhang W, Guo Z, et al. Elevated preoperative plasma D-dimer level is a useful predictor of chemoresistance and poor disease outcome for serous ovarian cancer patients. Cancer chemotherapy and pharmacology. 2015;76(6):1163-71.

18. Feng JF, Yang X, Chen S, Zhao Q, Chen QX. Prognostic Value of Plasma D-dimer in Patients with Resectable Esophageal Squamous Cell Carcinoma in China. Journal of Cancer. 2016;7(12):1663-7.

19. Chen WH, Tang LQ, Wang FW, Li CP, Tian XP, Huang XX, et al. Elevated levels of plasma D-dimer predict a worse outcome in patients with nasopharyngeal carcinoma. BMC cancer. 2014;14:583.

20. Inanc M, Er O, Karaca H, Berk V, Ozkan M, Dikilitas M, et al. D-dimer is a marker of response to chemotherapy in patients with metastatic colorectal cancer. Journal of BUON : official journal of the Balkan Union of Oncology. 2013;18(2):391-7

21. Pan JJ, Ng WT, Zong JF, Chan LL, O'Sullivan B, Lin SJ, et al. Proposal for the 8th edition of the AJCC/UICC staging system for nasopharyngeal cancer in the era of intensity-modulated radiotherapy. Cancer. 2016;122(4):546-58.

22. He SS, Wang Y, Yang L, Chen HY, Liang SB, Lu LX, et al. Plasma Fibrinogen Correlates with Metastasis and is Associated with Prognosis in Human Nasopharyngeal Carcinoma. Journal of Cancer. 2017;8(3):403-9.

23. Wojtukiewicz MZ, Sierko E, Rak J. Contribution of the hemostatic system to angiogenesis in cancer. Seminars in thrombosis and hemostasis. 2004;30(1):5-20.

24. Blom JW, Doggen CJ, Osanto S, Rosendaal FR. Malignancies, prothrombotic mutations, and the risk of venous thrombosis. Jama. 2005;293(6):715-22.

25. Ruf W, Mueller BM. Thrombin generation and the pathogenesis of cancer. Seminars in thrombosis and hemostasis. 2006;32 Suppl 1:61-8.

26. Klepfish A, Greco MA, Karpatkin S. Thrombin stimulates melanoma tumor-cell binding to endothelial cells and subendothelial matrix. International journal of cancer. 1993;53(6):978-82.

27. Zain J, Huang YQ, Feng X, Nierodzik ML, Li JJ, Karpatkin S. Concentration-dependent dual effect of thrombin on impaired growth/apoptosis or mitogenesis in tumor cells. Blood. 2000;95(10):3133-8.

28. Wojtukiewicz MZ, Tang DG, Ciarelli JJ, Nelson KK, Walz DA, Diglio CA, et al. Thrombin increases the metastatic potential of tumor cells. International journal of cancer. 1993;54(5):793-806

29. Franco-Chuaire ML, Magda Carolina SC, Chuaire-Noack L. Epithelial-mesenchymal transition (EMT): principles and clinical impact in cancer therapy. Investigacion clinica. 2013;54(2):186-205.

30. Gutierrez-Rodriguez M, Herranz R. From Multiple PAR1 Receptor/Protein Interactions to their Multiple Therapeutic Implications. Current topics in medicinal chemistry. 2015;15(20):2080-114

31. Song JS, Kang CM, Park CK, Yoon HK. Thrombin induces epithelial-mesenchymal transition via PAR-1, PKC, and ERK1/2 pathways in A549 cells. Experimental lung research. 2013;39(8):336-48.

32. Even-Ram S, Uziely B, Cohen P, Grisaru-Granovsky S, Maoz M, Ginzburg Y, et al. Thrombin receptor overexpression in malignant and physiological invasion processes. Nature medicine. 1998;4(8):909-14.

33. Liu Y, Mueller BM. Protease-activated receptor-2 regulates vascular endothelial growth factor expression in MDA-MB-231 cells via MAPK pathways. Biochemical and biophysical research communications. 2006;344(4):1263-70.

34. Asanuma K, Wakabayashi H, Okamoto T, Asanuma Y, Akita N, Yoshikawa T, et al. The thrombin inhibitor, argatroban, inhibits breast cancer metastasis to bone. Breast cancer (Tokyo, Japan). 2013;20(3):241-6.

35. Liu DQ, Li FF, Jia WH. Cumulative scores based on plasma D-dimer and serum albumin levels predict survival in esophageal squamous cell carcinoma patients treated with transthoracic esophagectomy. Chinese journal of cancer. 2016;35:11. 